\section{SCIENCE CHINA \\ Physics, Mechanics \& Astronomy}

\title{
Tailoring magnetism in semiconductors
}

\author{
Igor Žutić* and Tong Zhou \\ Department of Physics, University at Buffalo, State University of New York, Buffalo NY 14260, USA \\ Received February 7, 2018; accepted February 13, 2018; published online April 2, 2018
}

\begin{tabular}{ll}
\hline Citation: & I. Žutić, and T. Zhou, Tailoring magnetism in semiconductors, Sci. China-Phys. Mech. Astron. 61, 067031 (2018), https://doi.org/10.1007/s11433- \\
& $018-9191-0$
\end{tabular}

While logic devices in conventional electronics rely on the control of the charged carriers in the semiconductor chips, robust information storage in computer hard drives and magnetic random access memory is implemented using the carriers' spin in ferromagnetic metals. A long quest to realize magnetic semiconductors [1-3] can be simply summarized as: $1+1>2$, in hope that combining the control of charge and spin in a single material would become more than just the sum of its separate parts (charge and spin) and enable new or improved functionalities [3]. Indeed, there are intriguing opportunities in the class of dilute magnetic semiconductors (DMS) where by adding magnetic impurities, typically Mn, to a nonmagnetic semiconductor host it is possible to realize carrier-mediated magnetism [2,3]. DMS offer a control of the exchange interaction by tuning the ferromagnetic Curie temperature, $T_{\mathrm{c}}$, through changes in the carrier density, by an applied electric field and photoexcitation [2-5].

However, despite decades of research, the two most studied DMS classes of II-VI and III-V compounds [1-3] perhaps are better described as: $1+1<1$. Their individual charge and magnetic properties are not approaching those from better nonmagnetic semiconductors or ferromagnetic metals, respectively. In the II-VI DMS $\mathrm{Mn}^{2+}$ is isovalent with the group II ions and provides only spin doping; the corresponding lack of carriers makes ferromagnetism limited to only a few K [1-3]. In (Ga,Mn)As, the most common III-V DMS, $\mathrm{Mn}^{2+}$ leads to both spin and carrier doping enabling a higher $T_{\mathrm{c}}$ of up to $\sim 190 \mathrm{~K}$ [2], but limited by a low $\mathrm{Mn}^{2+}$

*Corresponding author (email: zigor@buffalo.edu) solubility which complicates its growth and can create nanoscale clustering of Mn ions. Instead of the desired single phase $(\mathrm{Ga}, \mathrm{Mn}) \mathrm{As}$, the outcome of the growth could yield nonmagnetic semiconductor GaAs accompanied by nanoclusters of ferromagnetic metal MnAs having a magnetic signal mistaken with (Ga,Mn)As [3]. This dual role of Mn as both spin and carrier doping creates a strong perturbation to the nonmagnetic host, significantly degrading the optical properties of GaAs and reducing its mobility by 2-3 orders of magnitude [3].

The discovery of the new II-II-V DMS class, such as (Ba, $\mathrm{K})(\mathrm{Zn}, \mathrm{Mn})_{2} \mathrm{As}_{2}$, referred to as BZA [6] revealed a promising path forward by providing an independent carrier $\left(\mathrm{K}^{+}\right)$and spin $\left(\mathrm{Mn}^{2+}\right)$ doping, solving several previous obstacles at once. There was no longer a lack of carriers, nor the low $\mathrm{Mn}^{2+}$ solubility allowing that the record $T_{\mathrm{c}}$ in $(\mathrm{Ga}, \mathrm{Mn}) \mathrm{As}$ is quickly overtaken by BZA, reaching $230 \mathrm{~K}$ [7]. With a moderate host perturbation by this independent doping desirable properties of the magnetic host are better preserved, enabling also a more quantitative description of the underlying origin of the magnetism in BZA $[8,9]$ than in $(I I I, M n) \mathrm{V}$ where it still remains debated $[2,10]$.

Despite these advances, the prior BZA research posed an important constraint: all the samples formed as a powder were polycrystalline, preventing many exciting developments in which BZA could be a parts of high-quality heterostructures that combine materials of very diverse properties [6]. In this context, the latest work of Zhao et al. [11] demonstrates an important breakthrough; not only does it realize the first single crystal BZA, but through a fabri- 
cation of superconductor/BZA heterostructure extracts the degree of carrier spin polarization, one of the key figure of merit for DMS and other magnets [3]. Probing this spin polarization was made possible by the process of Andreev reflection through conductance measurements.

To better understand the implication of this scattering process, we first consider a simple case of nonmagnetic region/superconductor (N/S) interface [12]. Figure 1(a) depicts the specular (ordinary) reflection, similar to a ball bouncing of a wall. In this case an electron (shown as the solid circle) approaching the N/S interface is reflected with the same charge ("e") and the same spin ("up arrow'). No electrical current is transferred to the $\mathrm{S}$ region and there is no contribution to the measured conductance, $G$. In contrast, during Andreev reflection, depicted in Figure 1(b), an electron approaching the interface is reflected backwards and converted into a hole (the absence of an electron is depicted as an empty circle) with opposite charge and spin. From the charge conservation we could infer that two electron charges are transferred across the interface into the $\mathrm{S}$ region. These two electrons with opposite spins form a Cooper pair and are responsible for doubling of the normal state conductance, $G=2 G_{0}$. Analogous considerations apply also for an incident hole. For N/S heterostructures there is a widely used BTK picture [12] to describe charge transport. The interfacial scattering is modeled by a delta function having a strength $Z$, related to the normal state transmission coefficient as $T=1 /(1$ $+Z^{2}$ ). $Z \gg>1$ implies a tunnel contact and vanishing conductance dominated by the specular reflection. While in the opposite limit, $Z<<1$, the contact is highly transparent, specular reflection is suppressed and each incoming particle can undergo Andreev reflection to yield $G=2 G_{0}$. This picture of an equal spin up/down population allows neglecting spin degrees of freedom, there is also no need to distinguish if $Z$ comes just from an intrinsic N/S barrier or if it has an additional contribution from the Fermi velocity mismatch, arising from different carrier density in the $\mathrm{N}$ and $\mathrm{S}$ region.

For DMS/S heterostructure studied by Zhao et al. [11] the situation is more complex and theoretical picture is still evolving, a single $Z$ parameter is not sufficient to describe the interfacial scattering $[13,14]$. However, the main trends for Andreev reflection and the measured bias-dependent conductance $G(V)$ from ref. [11] can already be inferred from Figure 1(c). By focusing on the low-bias conductance, below the superconducting gap, $|e V|<\Delta$, the exchange splitting in DMS leads to unequal density of states and spin-polarized carriers, with a polarization, $P$. Only a fraction of majority spin carriers can find a partner of opposite spin and contribute to $G$ through Andreev reflection. In a half-metal, a fully spin-polarized material, $P=100 \%$, this would imply vanishing $G$ at low bias. While suppressed $G$ would also be possible for $P=0$ if $Z>>1$. In that tunneling limit the Andreev reflection is negligible and the $G$ would resemble the density (a)

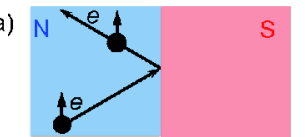

(b) $N$

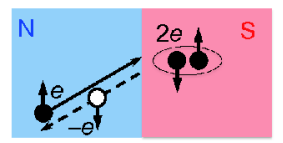

(c)

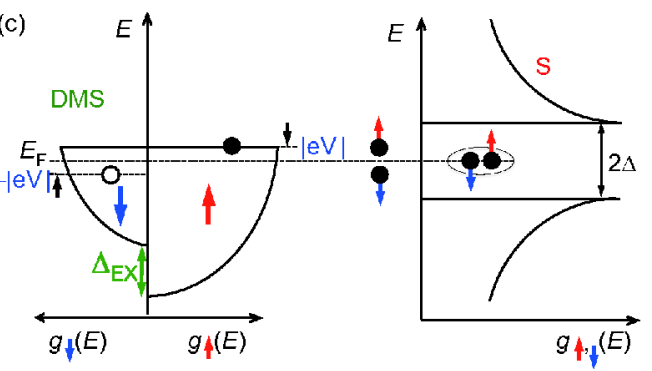

Figure 1 (Color online) Specular (a) and Andreev reflection (b) at the nonmagnetic region/superconductor (N/S) interface. (c) Andreev reflection at the DMS/S interface. DMS region has an exchange splitting and unequal density of states. Near the Fermi level, $E_{\mathrm{F}}$, only a fraction of incident electrons with spin up can find a partner of opposite spin and contribute to the charge transfer by entering the $\mathrm{S}$ region and forming a Cooper pair.

of states for the S region [12], shown in Figure 1(c), with pronounced peaks at applied bias corresponding to $\Delta$. However, since Zhao et al. have measured a strongly suppressed $G / G_{0}$ without such pronounced peaks, their measurements confirm that BZA is highly-spin polarized and that the interface is transparent with only a small $Z$. In fact, their given value of $P=66 \%$ already exceeds $P \sim 50 \%$ measured for NiMnSb, one of the first predicted half-metals $[3,15]$. A more general theoretical description of DMS/S including interfacial spin-flip and spin-orbit coupling (SOC), as well as the mismatch between the Fermi velocities of the DMS and $\mathrm{S}$ region may lead to even higher value of $P$ for BZA. $\mathrm{Pb}$ used as the $\mathrm{S}$ region is a well-known example of a material with a strong SOC.

This realization of a single crystal BZA with a large $P$ in a highly-transparent heterostructure by Zhao et al. opens several tantalizing prospects. In addition to probing carrier spin polarization, could Andreev reflection be used to probe SOC? In spintronics SOC has a crucial role by enabling electric control of magnetization and, conversely, a magnetic control of electric current. In systems without the space inversion symmetry, SOC induces spin-orbit fields responsible for a number of fundamental phenomena that are absent or fragile in the bulk, such as the tunneling magnetoanisotropy, skyrmions, and spin-orbit torques [14]. Moreover, predicted sensitivity of Andreev reflection to the direction of magnetization in DMS may lead to SOC manifestation that are enhanced as compared to the corresponding normal state phenomena [14]. Could the temperature window for probing $P$ or SOC be enhanced by replacing $\mathrm{Pb}$, with high-temperature "122" type iron-based superconductor where $(\mathrm{Zn}$, $\mathrm{Mn})$ in BZA is replaced by Fe? Which other BZA-based heterostructures are possible? What is the limit for $T_{\mathrm{c}}$ in II-II$\mathrm{V}$ DMS? Can $T_{\mathrm{c}}$ be enhanced even above $300 \mathrm{~K}$ by substituting As in BZA with $\mathrm{Sb}$ or P [8]? 
As we await for the answers of these and many other questions stimulated by the latest advances of Jin and collaborators, it seems that the future of DMS is bright and we may not need to abandon yet a simple hope that $1+1>2$.

This work was supported by the U.S. Department of Energy, Office of Basic Energy Sciences (Grant No. DE-SC004890).

1 J. K. Furdyna, J. Appl. Phys. 64, R29 (1988).

2 T. Dietl, and H. Ohno, Rev. Mod. Phys. 86, 187 (2014), arXiv: 1307.3429.

3 I. Žutić, J. Fabian, and S. Das Sarma, Rev. Mod. Phys. 76, 323 (2004).

4 S. Koshihara, A. Oiwa, M. Hirasawa, S. Katsumoto, Y. Iye, C. Urano, H. Takagi, and H. Munekata, Phys. Rev. Lett. 78, 4617 (1997).

5 H. Ohno, D. Chiba, F. Matsukura, T. Omiya, E. Abe, T. Dietl, Y. Ohno, and K. Ohtani, Nature 408, 944 (2000).

6 K. Zhao, Z. Deng, X. C. Wang, W. Han, J. L. Zhu, X. Li, Q. Q. Liu, R. C. Yu, T. Goko, B. Frandsen, L. Liu, F. Ning, Y. J. Uemura, H. Dabkowska, G. M. Luke, H. Luetkens, E. Morenzoni, S. R. Dunsiger, A. Senyshyn, P. Böni, and C. Q. Jin, Nat. Commun. 4, 1442 (2013).
7 K. Zhao, B. Chen, G. Zhao, Z. Yuan, Q. Liu, Z. Deng, J. Zhu, and C. Jin, Chin. Sci. Bull. 59, 2524 (2014).

8 T. P. A. Hase, M. S. Brewer, U. B. Arnalds, M. Ahlberg, V. Kapaklis, M. Björck, L. Bouchenoire, P. Thompson, D. Haskel, Y. Choi, J. Lang, C. Sánchez-Hanke, and B. Hjörvarsson, Phys. Rev. B 90, 104403 (2014).

9 H. Suzuki, G. Q. Zhao, K. Zhao, B. J. Chen, M. Horio, K. Koshiishi, J. $\mathrm{Xu}$, M. Kobayashi, M. Minohara, E. Sakai, K. Horiba, H. Kumigashira, B. Gu, S. Maekawa, Y. J. Uemura, C. Q. Jin, and A. Fujimori, Phys. Rev. B 92, 235120 (2015), arXiv: 1509.04520.

10 M. Tanaka, S. Ohya, and P. Nam Hai, Appl. Phys. Rev. 1, 011102 (2014), arXiv: 1311.6616.

11 G. Q. Zhao, C. J. Lin, Z. Deng, G. X. Gu, S. Yu, X. C. Wang, Z. Z. Gong, Y. J. Uemera, Y. Q. Li, and C. Q. Jin, Sci. Rep. 7, 14473 (2017).

12 G. E. Blonder, M. Tinkham, and T. M. Klapwijk, Phys. Rev. B 25, 4515 (1982).

13 I. Žutić, and S. Das Sarma, Phys. Rev. B 60, R16322 (1999).

14 P. Högl, A. Matos-Abiague, I. Žutić, and J. Fabian, Phys. Rev. Lett. 115, 116601 (2015), arXiv: 1502.08022.

15 R. J. Jr. Soulen, J. M. Byers, M. S. Osofsky, B. Nadgorny, T. Ambrose, S. F. Cheng, P. R. Broussard, C. T. Tanaka, J. Nowak, J. S. Moodera, A. Barry, and J. M. D. Coey, Science 282, 85 (1998). 